\title{
Experimental Investigation on the Onset of Modulation Instability as a Precursor for Stimulated Brillouin Scattering in Yb-doped Fiber Amplifiers
}

\author{
Yusuf Panbiharwala ${ }^{\mathrm{a}}$, Aditi Ghosh ${ }^{\mathrm{a}}$, Johan Nilsson ${ }^{\mathrm{b}}$, Deepa Venkitesh ${ }^{\mathrm{a}}$, Balaji Srinivasan ${ }^{\mathrm{a}}$ \\ ${ }^{a}$ Department of Electrical Engineering, Indian Institute of Technology Madras, Chennai-600036; \\ ${ }^{b}$ Optoelectronics Research Center, University of Southampton, UK
}

\begin{abstract}
We report an experimental investigation on the onset of SBS-induced modulation instability in a CW Yb-doped fiber amplifier. Threshold for such backscattered signals is found to be well below SBS threshold.
\end{abstract}

Keywords: Fiber lasers and amplifiers, stimulated Brillouin scattering, SBS Dynamics, Yb-doped fiber amplifiers

\section{INTRODUCTION}

High power fiber laser sources have numerous applications in material processing, medical surgery and defense. The master oscillator power amplifier (MOPA) scheme is usually a reliable and scalable approach towards developing such sources ${ }^{1}$. Unfortunately scaling to high powers is limited by nonlinear processes such as stimulated Brillouin scattering (SBS), especially when the source linewidth is narrow. Specifically, the onset of SBS can generate intense pulses in the backward direction, which can cause severe damage to upstream components ${ }^{2}$. SBS is reported to have stochastic or chaotic dynamic behavior near threshold ${ }^{3-6}$. Such behavior is also referred to as SBS induced modulation instability, which can have a threshold lower than the conventional Brillouin threshold ${ }^{7}$. Hence, a systematic monitoring of backscattered pulses can potentially serve as a precursor and thus early warning of impending catastrophic damage due to SBS and thereby aid power scaling of Yb-doped fiber laser sources in a reliable manner.

In this work, we present an investigation of such backscattered pulses due to SBS in a continuous-wave (CW) MOPA source based on Yb-doped silica fiber. We detect seemingly chaotic pulses much below the SBS threshold, which can possibly act as a precursor to SBS. We also report the dynamics of the pulse evolution near the SBS threshold in absence of reflections from the output end.

\section{EXPERIMENTAL SETUP AND RESULTS}

The schematic of the experimental setup of the three-stage amplifier in the MOPA configuration is shown in Figure 1. The seed is a Fabry Perot (FP) semiconductor diode laser (Lumics model \#LU1064M300), which is wavelength stabilized at $1064 \mathrm{~nm}$ by a Fiber Bragg Grating (FBG) spliced $4 \mathrm{~cm}$ away from the diode. The signal power of $\sim 36 \mathrm{~mW}$ from the laser diode is amplified to $\sim 100 \mathrm{~mW}$ by Fiber Amplifier 1 (FA1), which comprises $1.5 \mathrm{~m}$ of $\mathrm{Yb}$-doped single clad fiber and is pumped at $975 \mathrm{~nm}$. The output of FA1 is amplified to $\sim 2 \mathrm{~W}$ by a second amplifier stage (FA2) comprising $5 \mathrm{~m}$ of Yb-doped double clad fiber (core/clad diameter $-10 / 125 \mu \mathrm{m}$ ) and cladding-pumped at $915 \mathrm{~nm}$. The output of FA2 is further amplified in a third amplifier stage (FA3), which is built with $3.3 \mathrm{~m}$ of Yb-doped double clad fiber (core/clad diameter 15/125 $\mu \mathrm{m}$ ) and cladding-pumped at $975 \mathrm{~nm}$. In order to protect the seed source from any backward propagating signal and to monitor this signal, we employ mid-stage isolators with output tap ports $(0.05 \%)$. Time domain measurements using fast photo-detector at backward tap ports of input-end isolator are done to detect the backscattered pulses. The entire MOPA assembly is fusion spliced and the fiber at the output of FA3 stage $(\sim 2 \mathrm{~m}$ undoped fiber which is spliced to remove un-absorbed pump power) is maintained with an angle cleave ( 12 degree) in order to avoid any spurious reflections. 


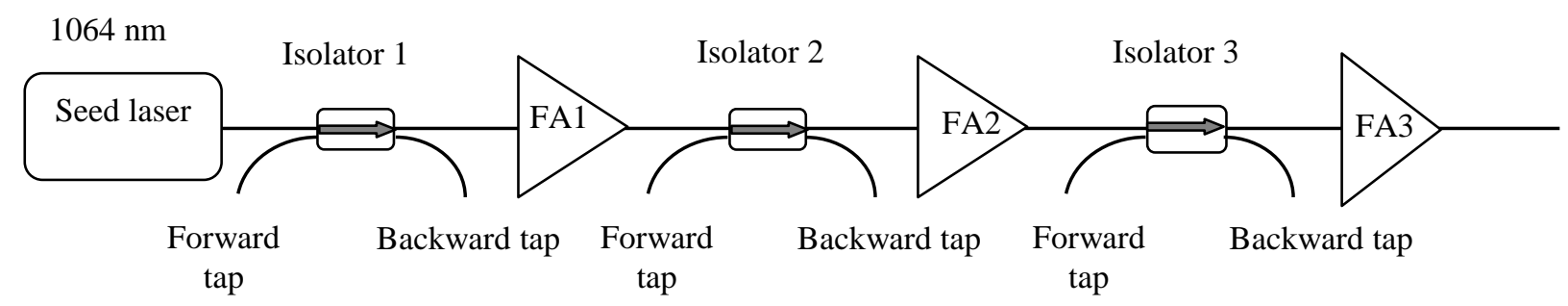

Figure 1: Experimental setup for monitoring the forward and backward propagating signals.

The output powers from FA1 and FA2 are $100 \mathrm{~mW}$ and $2 \mathrm{~W}$ respectively. Figure 2a shows the increase in output signal power with pump power for FA3. No pulsing is observed when the signal power from FA3 is below $16 \mathrm{~W}$. As the pump power is further increased we observe pulsing behavior in the backward direction. Figure $2 \mathrm{~b}$ and $2 \mathrm{c}$ show FA3 backward power fluctuations for an output power of $13 \mathrm{~W}$ and $16 \mathrm{~W}$ respectively. We observe that the pulses occur in bursts (pulsing ON and OFF state) and the duration of their occurrence and the pulse amplitude increases as the signal is amplified further. Such pulsing has been reported to occur as modulation instability due to $\mathrm{SBS}^{7}$ and similar occurrence in the form of bursts has also been reported ${ }^{5}$. A key requirement for the onset of the SBS at these power levels is that the source linewidth is narrow.
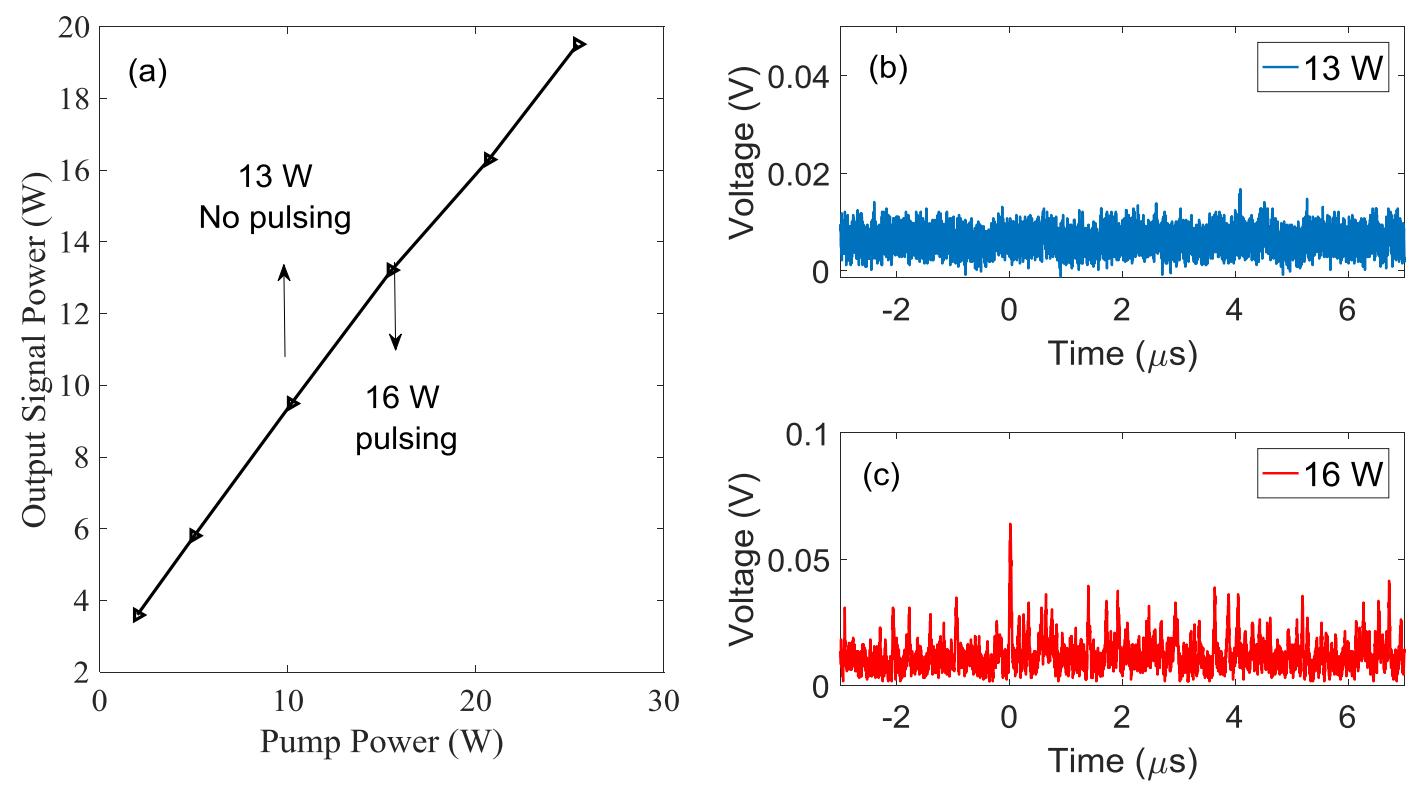

Figure 2: (a) Output power of the FA3 amplifier stage as a function of the pump power. (b) and (c) Time domain traces captured for the backscattered pulses using $400 \mathrm{MHz}$ photodetector (Thorlabs) at $13 \mathrm{~W}$ and $16 \mathrm{~W}$ of signal power.

\section{ANALYSIS AND DISCUSSION}

Our seed laser is a Fabry-Perot (FP) semiconductor diode laser which is wavelength stabilized at $1064 \mathrm{~nm}$ by a FBG and whose 3-dB linewidth is specified as $0.6 \mathrm{~nm}$ by the manufacturer. We measure 3 - $\mathrm{dB}$ linewidth as $0.07 \mathrm{~nm}$, which is limited by the resolution of optical spectrum analyzer (OSA). To improve the measurement resolution of the spectrum, we then performed the linewidth measurement using delayed self-heterodyne setup ${ }^{8}$ whose output is captured using an electronic spectrum analyzer (ESA, Rhode and Schwarz FSV series) as shown in Figure 3a. In order to remove the effect of source coherence in the heterodyne signal, we use $25 \mathrm{~km}$ (which gives resolution of $\sim 8 \mathrm{kHz}$ ) long standard telecom 
fiber in one arm of the interferometer. We also implement a frequency shifter in the other arm using an acousto-optic modulator (AOM) so that the beating between the signals of the two arms occurs at a shifted frequency of $200 \mathrm{MHz}$. The component centered at $200 \mathrm{MHz}$ on electrical spectrum analyzer (ESA) shows the 3-dB linewidth of $\sim 100 \mathrm{kHz}$ (Figure $3 b)$. Since the observed linewidth from such a delayed heterodyne setup is twice the laser linewidth, we deduce the laser linewidth as $50 \mathrm{kHz}$.

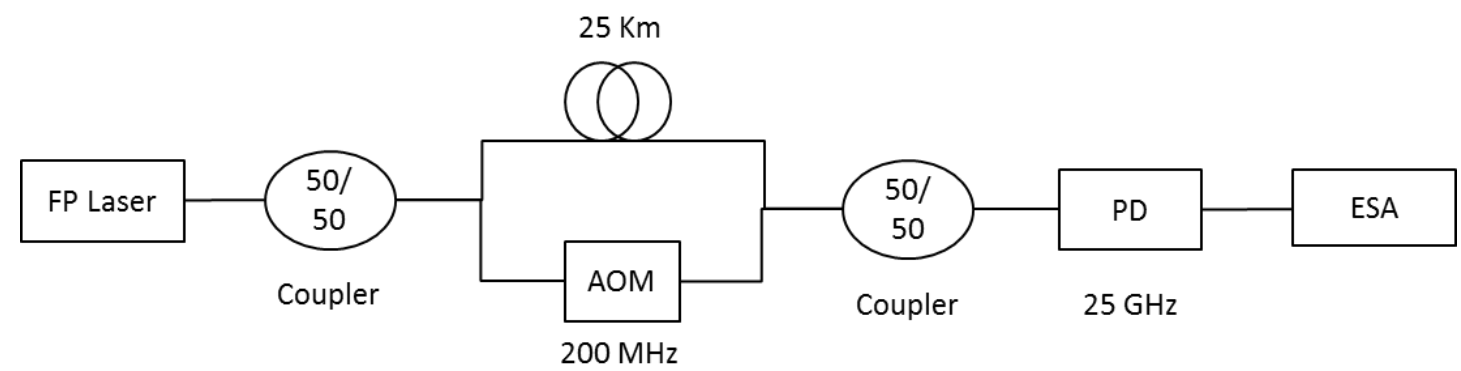

(a)

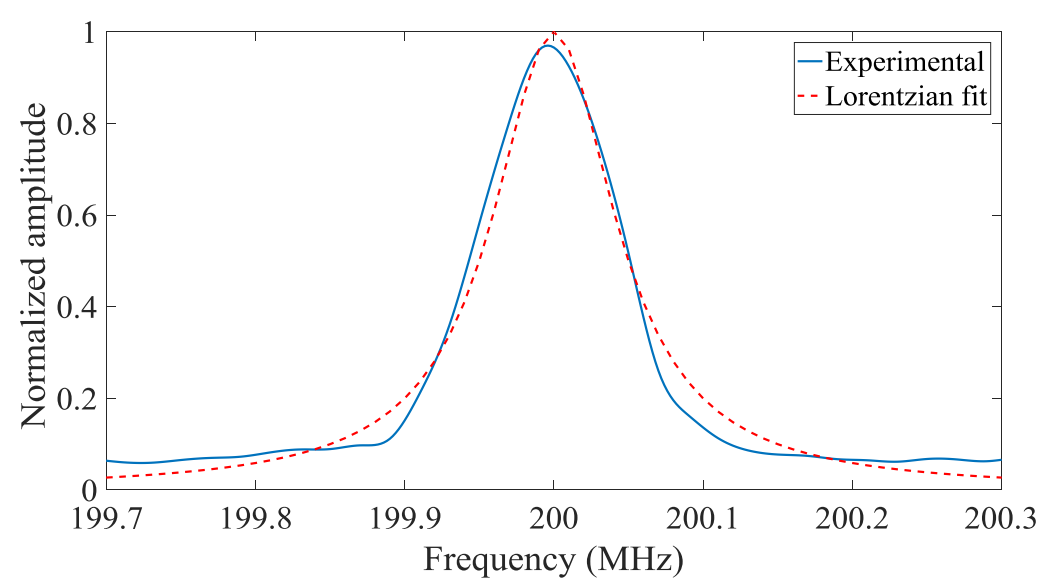

(b)

Figure 3: (a) Schematic of the experimental setup used to measure the seed laser linewidth using the delayed self-heterodyne method, (b) Spectrum measured on electrical spectrum analyzer (ESA) and the corresponding Lorentzian fit.

Since the FBG is spliced at $4 \mathrm{~cm}$ away from the diode facet, reflections from this FBG could result in multiple longitudinal modes spaced at $2.5 \mathrm{GHz}$ in the output electrical spectrum. We attempted to measure such high frequency spectral content of the heterodyne signal using a high speed photodetector (Newport, $25 \mathrm{GHz}$ ). We observed that no such higher frequency content were detectable, implying that the FP laser is effectively operating with a single longitudinal mode of linewidth $50 \mathrm{kHz}$ at any given instant of time.. Such single longitudinal mode operation of our seed laser diode may indeed result in the SBS-induced modulation instability discussed above.

The other key aspect of any SBS-induced modulation instability is the manifestation of a threshold behavior at higher power levels. In order to quantify such a threshold for SBS, we observed the average backscattered power for various values of output signal power from FA3 and deduced the SBS reflectivity which is defined as the ratio of backscattered power to the output signal power ${ }^{9-10}$. Figure 4 illustrates the plot of SBS reflectivity as a function of forward signal power at $1064 \mathrm{~nm}$. We observe that the SBS reflectivity indeed shows a dramatic increase beyond a certain signal power. It has been reported previously that the SBS threshold can be defined for any value of reflectivity between $0.1 \%$ to $10 \%$ as the reflectivity increases exponentially around the threshold region ${ }^{9}$. We define the SBS threshold at the reflectivity of $0.5 \%$, which in our system corresponds to an output signal power value of $30.3 \mathrm{~W}$. In order to confirm that the FP laser exhibits single longitudinal mode oscillation, we also performed similar experiment with a DFB based diode laser 
(Eagleyard photonics \#EYP-DFB-1064), which has a manufacturer defined linewidth of $2 \mathrm{MHz}$. We measured the SBS threshold for DFB laser to be $29.4 \mathrm{~W}$ at $0.5 \%$ reflectivity, which is almost same as that of FP laser.

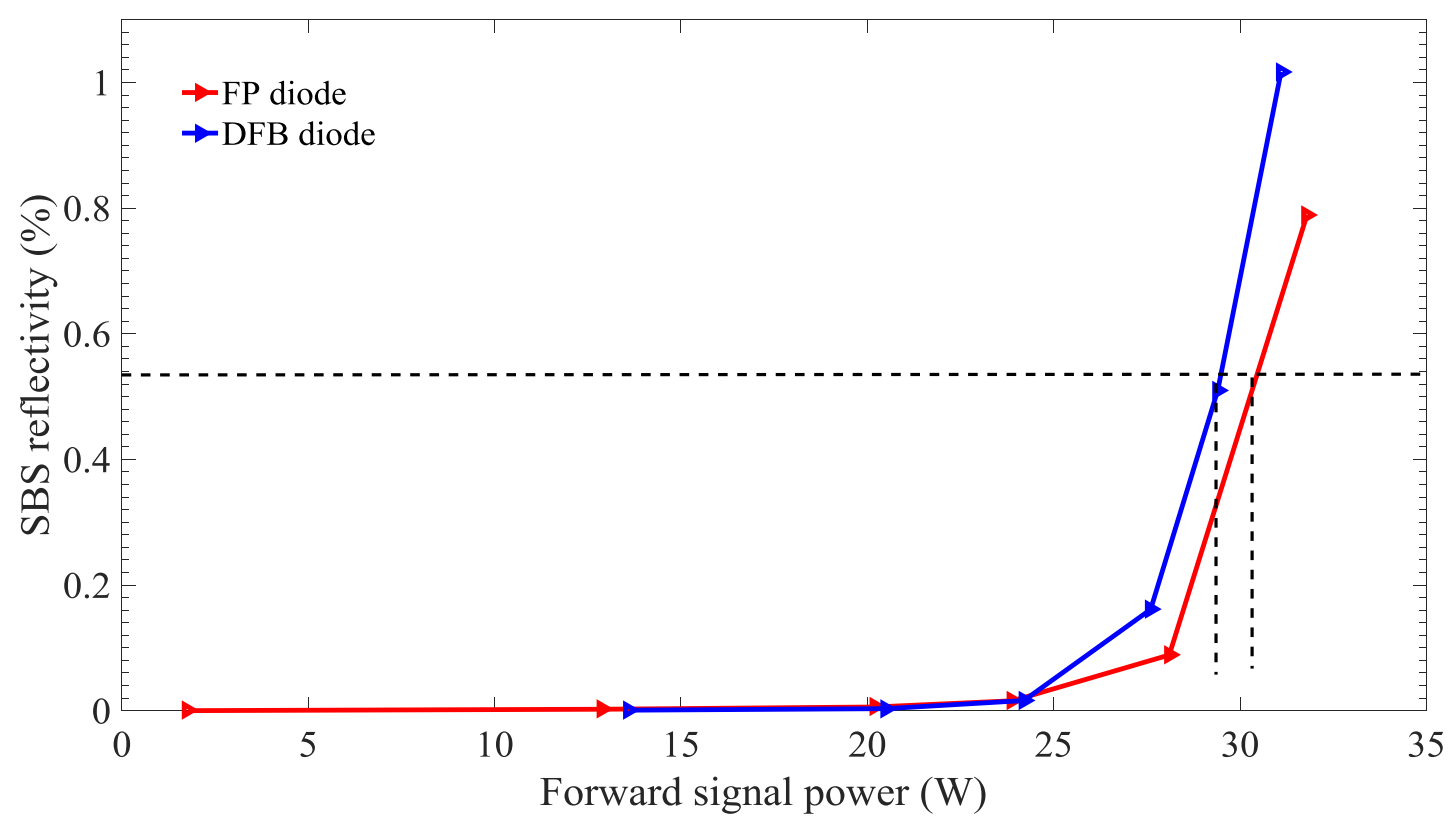

Figure 4: Plot of the SBS reflectivity as a function of the forward signal power for the FP and DFB lasers. The plot shows that the two lasers exhibit similar SBS threshold values.

As mentioned in the Introduction, a key objective of our work is to observe the SBS-induced backscattered power and possibly use it as a precursor for the onset of SBS itself. To this end, we investigated the pulsing behavior observed with the FP diode for different signal power levels. It has been previously reported that the backscattered pulses due to SBS exhibit a periodic pattern beyond the SBS threshold ${ }^{6}$. An elegant approach to establish the periodicity of the backscattered pulses is to plot the phase portrait of the captured data, which is obtained by phase space reconstruction from the time series data. In such a reconstruction, the amplitude of the pulses are plotted with respect to a time-delayed version of itself. A key aspect of such phase portraits is the precise determination of the time delay. Such precise determination is also possible from observing the first null crossing (i.e. zero correlation point) of the autocorrelation $\mathrm{C}(\tau)$. We determine $\mathrm{C}(\tau)$ by computing the Pearson correlation coefficient ${ }^{11}(\mathrm{r})$ which is defined below for all the value of $\tau$ (delay).

$$
r=\frac{\sum_{i}\left(x_{i}-\bar{x}\right)\left(y_{i}-\bar{y}\right)}{\sqrt{\sum_{i}\left(x_{i}-\bar{x}\right)^{2}} \sqrt{\sum_{i}\left(y_{i}-\bar{y}\right)^{2}}}
$$

Where $x_{i}$ represents the captured data, $y_{i}$ represents delayed version (by $\tau$ ) of the same data, $\bar{x}$ and $\bar{y}$ represents the mean of $x_{i}$ 's and $y_{i}$ 's respectively.

Let us consider the case of the time dynamics captured for 0.66 times SBS threshold $\left(0.66 P_{t h}{ }^{S B S}\right)$, i.e. at $20 \mathrm{~W}$ of output signal power. We capture the time dynamics for $100 \mu \mathrm{s}$ duration, from which a sample slice of $20 \mu \mathrm{s}$ duration is shown in Figure 5a. It has been reported earlier ${ }^{6}$ that the backscattered pulses for an output signal power just above the SBS threshold exhibit a pulse spacing corresponding to the round trip time along the fiber. At the $20 \mathrm{~W}$ power level, we 
did not observe any such pulses spaced at $\sim 70$ ns (round trip time for our case). In fact, the pulses appear much broader, possibly due to bunching of multiple successive pulses.

The autocorrelation of the captured pulses for $100 \mu \mathrm{s}$ is shown in Figure 5b. The first null of the autocorrelation occurs at a time delay of $\sim 0.22 \mu \mathrm{s}$. Using this time delay, we constructed the corresponding phase portrait as shown in Figure 5c. The phase portrait does not show any obvious structures. However, we notice that the structure of the autocorrelation plot is significantly wider compared to that obtained for lower signal power levels $(13 \mathrm{~W})$. Specifically, the observation of a larger width of the autocorrelation peak indicates that there is a certain level of deterministic pulsing even at $0.66 P_{t h}{ }^{S B S}$. It is to be noted that we applied a low pass filter of $100 \mathrm{MHz}$ bandwidth to the above time series in order to eliminate undesirable noise from the captured time series leading to smoother phase portraits. The bandwidth of the applied filter is chosen such that it is larger than the inverse of the round-trip time ( $70 \mathrm{~ns})$ for FA3. We have verified that the application of the above low pass filter does not modify the autocorrelation trace significantly.

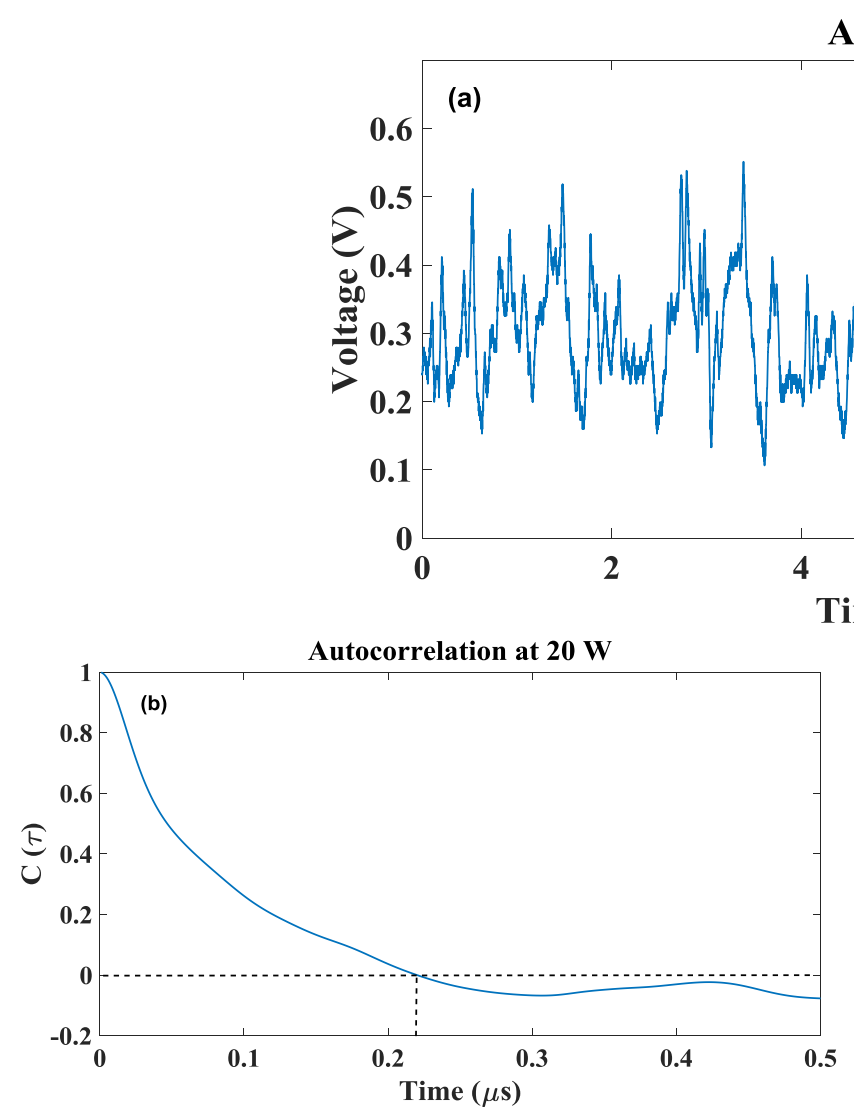

At 20 W
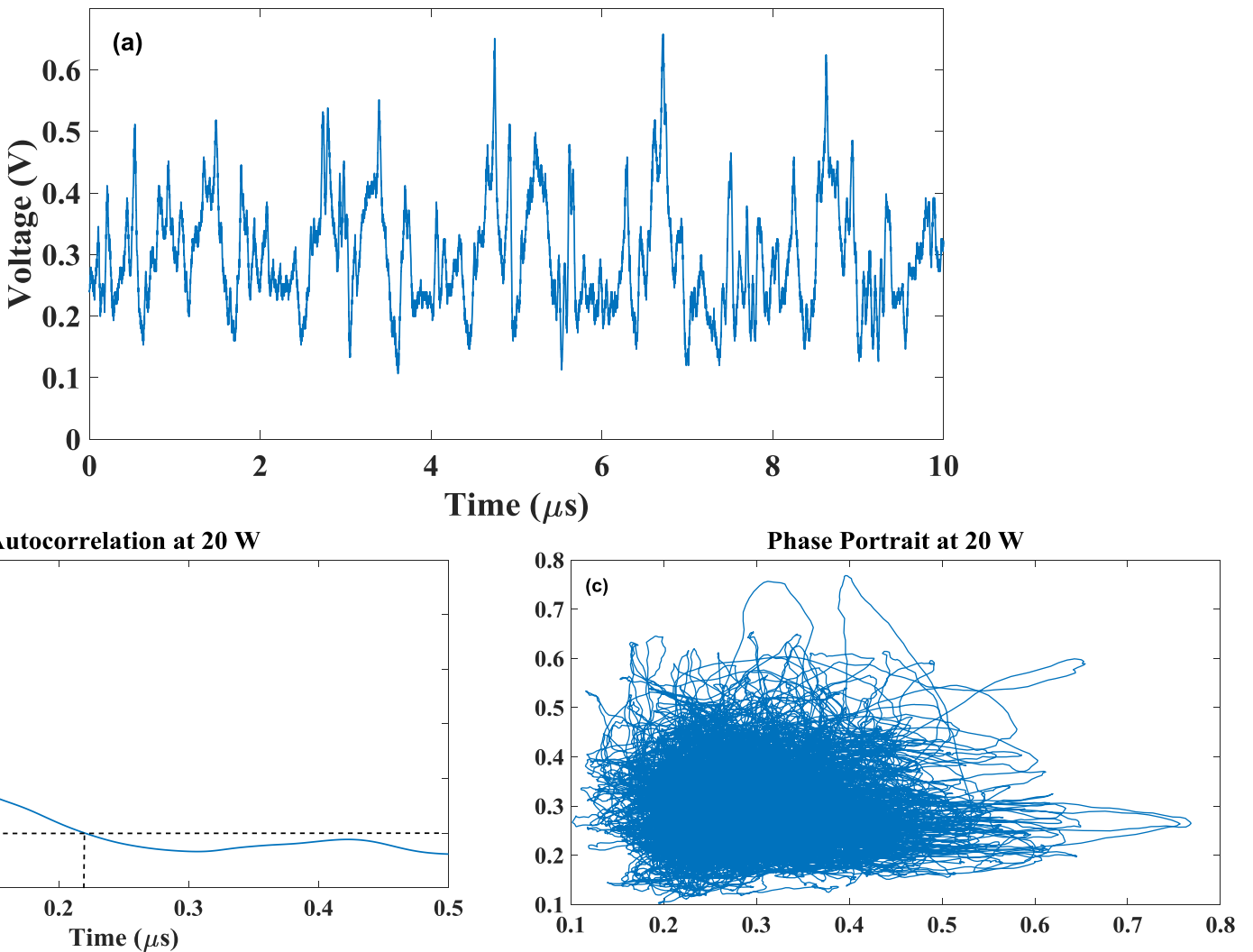

Figure 5: (a) Time trace captured at a forward signal power of $20 \mathrm{~W}$ and the corresponding (b) autocorrelation and (c) phase portraits of time series

We next consider the time traces at higher power levels of $24 \mathrm{~W}\left(0.8 P_{t h}{ }^{S B S}\right)$ and $31.8 \mathrm{~W}\left(1.1 P_{t h}{ }^{S B S}\right)$. A sample time slice of the backscattered trace captured at the above power levels are shown in Figs. 6a and 6b. We observe that some pulses having pulsewidth approximately corresponding to the round trip time of FA3 starts occurring clearly as compared to pulses observed at $20 \mathrm{~W}$ power levels. In fact, we see a clear periodic pulse train at $31.8 \mathrm{~W}$ signal power, whose pulsewidth corresponds nearly to the round trip time of FA3.

The autocorrelation plot for the time traces obtained at the above power levels are shown in Figs. 6c and 6d. Compared to the autocorrelation trace obtained at $20 \mathrm{~W}$ signal power, we observe that the FWHM of the autocorrelation plot has reduced. Specifically, we clearly see a periodic autocorrelation trace with damped oscillation for the $31.8 \mathrm{~W}$ 
signal power which is consistent with the periodic pulse train observed in the time trace. Similar behavior in autocorrelation plots above SBS threshold with increasing gain have been reported earlier ${ }^{4}$.

Finally, based on the null point of the above autocorrelation traces we plot the phase portrait for the two different signal power levels as shown in Figs. 6e and 6f. While the phase portrait for the $24 \mathrm{~W}$ signal power is still not exhibiting any discernable structure, the phase portrait for $31.8 \mathrm{~W}$ clearly exhibits multiple loops (shown in inset of Fig. 6f) which are indicative of the periodic train of pulses.
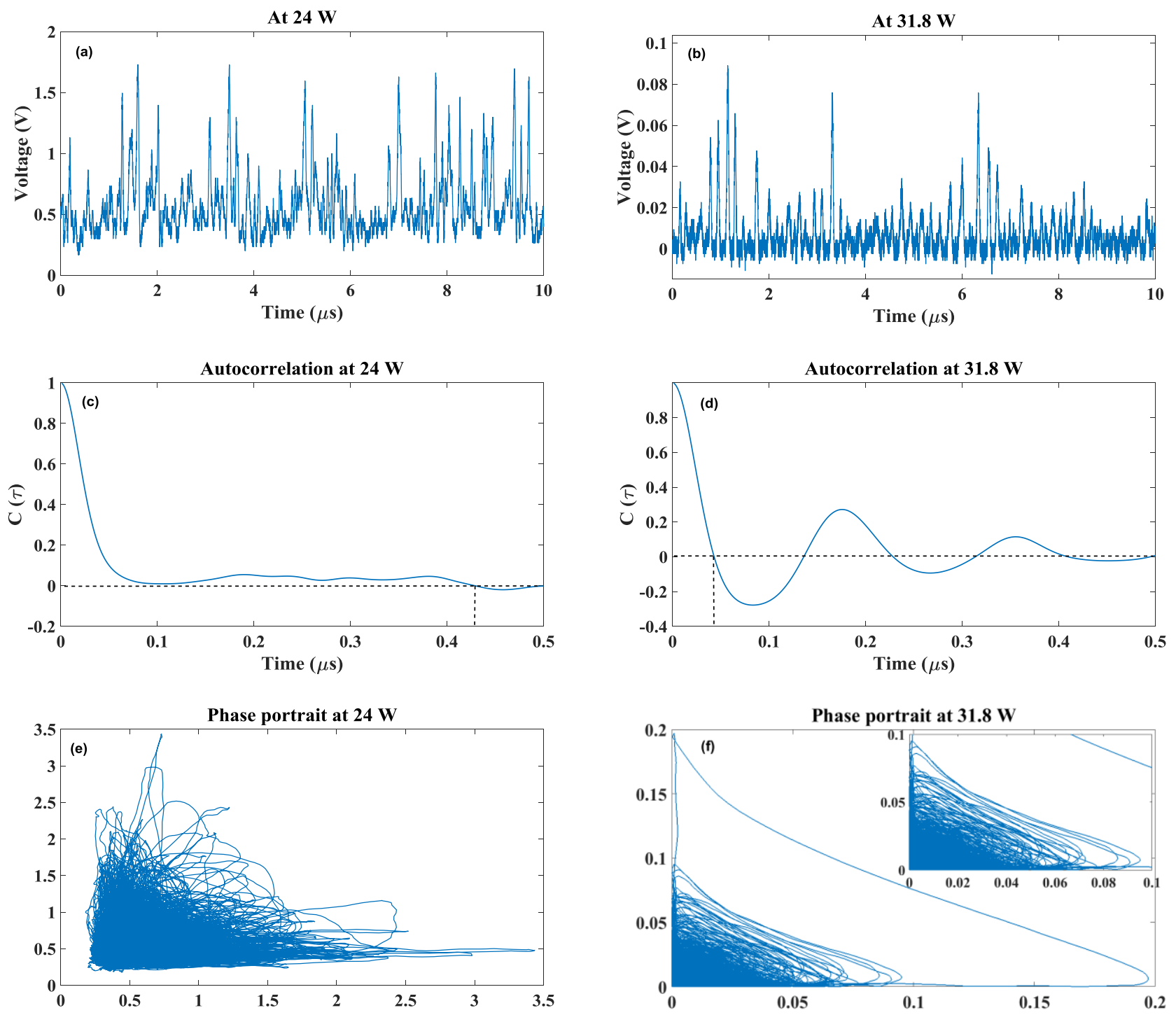

Figure 6: (a) and (b) shows time trace, (c) and (d) shows autocorrelation plot and (e) and (f) shows phase portraits corresponding to $28 \mathrm{~W}$ and $31.8 \mathrm{~W}$ of forward signal power respectively

The FWHM of autocorrelation plot for the pulses captured at various power levels is plotted (Figure 7). We observe a clear transition as the pulses start forming at $20 \mathrm{~W}$ as compared to no pulsing at lower signal power levels (noise traces whose autocorrelation looks close to an impulse function). Such a signature is a clear indicator of the SBS-induced modulation instability, which is exhibited at a power level corresponding to $0.66 P_{t h}{ }^{S B S}$ itself. As such, the pulses monitored at the backward port of amplifier can give clear indication of the onset of SBS thereby acting as a precursor. 
This early detection is particularly useful in high power fiber amplifiers expected to operate near the SBS threshold with parameters that may vary (e.g., the pump wavelength drift). The SBS threshold can then be exceeded (e.g., as a result of an increased effective length), which can result in damage of various optical components. We also observe a transition in FWHM of autocorrelation at $24 \mathrm{~W}$ where we observe initiation of round trip pulse formation. Then as we go above SBS we do not observe any change in the FWHM of the autocorrelation but the autocorrelation of the data clearly start showing damped oscillation kind of behavior.

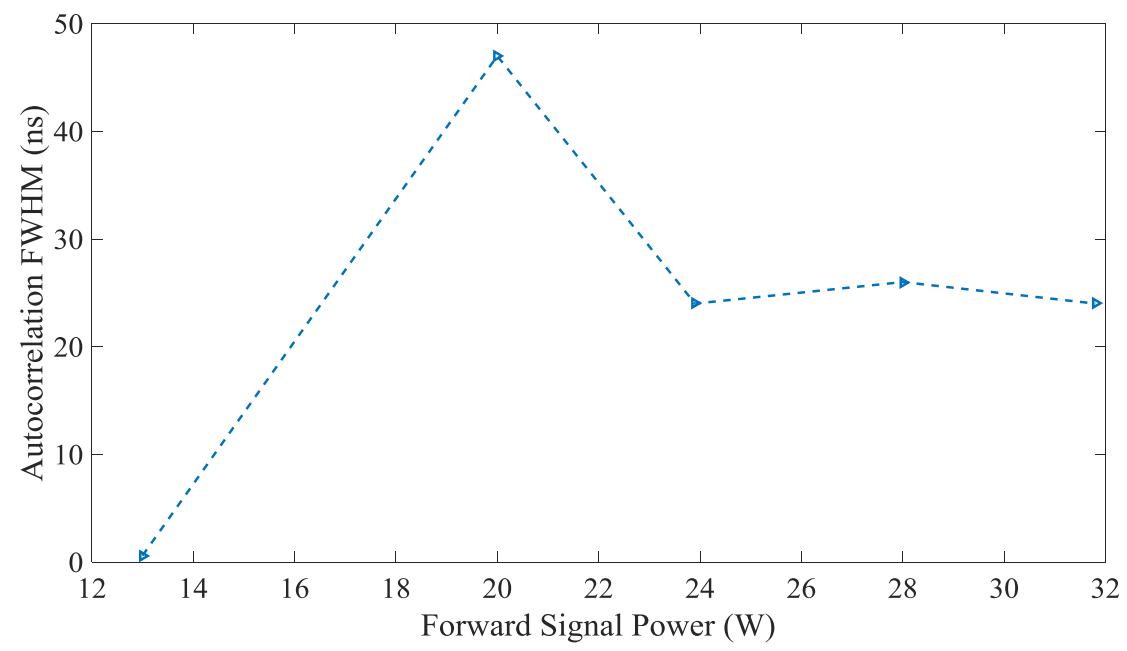

Figure 7: FWHM of autocorrelation for pulses captured at various forward signal power levels

\section{CONCLUSION}

We experimentally investigated the occurrence of SBS-induced modulation instability in Yb-doped fiber amplifier. The onset of the instability is observed due to the narrow linewidth of the source laser and the threshold for its occurrence is found to be below the SBS threshold. Through a careful study of the autocorrelation and phase portrait of the time series data, we establish the detection of the onset of SBS at a level as low as $0.66 \mathrm{P}_{\mathrm{th}}{ }^{\mathrm{SBS}}$. Such early detection of pulsing in backward direction and appropriate reduction in the pump power can serve as a precursor for the onset of SBS and help prevent catastrophic damage of components in MOPA-based fiber laser sources.

\section{Acknowledgements}

The authors would like to acknowledge Air Force Office of Scientific Research Grant (FA2386-15-1-5044) for funding and Visvesvaraya Scholarship, Government of India for supporting Yusuf's doctoral research. We would also like to acknowledge technical support from Suresh Kumar and Sathish Kumar in carrying out the experiments.

\section{REFERENCES}

[1] D. J. Richardson, J. Nilsson, and W. A. Clarkson," High power fiber lasers: current status and future perspectives [Invited],” Opt. Soc. Am. B 27, B63-B92 (2010).

[2] M. Melo, M. O. Berendt, J. M. Sousa, "Destructive random backscattering pulses showing Brillouin signature in MOPA fiber laser systems", Proc. of SPIE, 8601, 86011P (2013)

[3] R. G. Harrison, J. S. Uppal, A. Johnstone, and J. V. Moloney, "Evidence of Chaotic Stimulated Brillouin Scattering in Optical Fibers," Physical review letters 65 (2), 167-171 (1990).

[4] Alexandra L. Gaeta and Robert W. Boyd, "Stochastic dynamics of Stimulated Brillouin Scattering in an optical Fiber," Physical Review A, 44(5), 3205-3209 (1991) 
[5] Sang Hun Lee, Chil-Min Kim," Chaotic stimulated Brillouin scattering near the threshold in a fiber," Optics letters, 31(21), 3131-3133 (2006)

[6] R.G. Harrison, Dejin Yu, Weiping Lu, P.M. Ripley,"Chaotic stimulated Brillouin scattering: theory and experiment," PhysicaD, 86, 182-188 (1995).

[7] Govind P. Agrawal, [Nonlinear Fiber Optics], $4^{\text {th }}$ edition, Academic Press, Elsevier, (2007).

[8] Dennis Derickson, [Fiber optic test and measurement], Hewlett-Packard Company, Prentice Hall PTR, New Jersey, (1998).

[9] Andrey Kobyakov, Michael Sauer and Dipak Chowdhury, "Stimulated Brillouin scattering in optical fibers," Advances in Optics and Photonics 2, 1-59 (2010)

[10] Kyung-Hyun Lee et. al., "Transverse mode instability induced by stimulated Brillouin scattering in a pulsed singlefrequency large-core fiber amplifier," Applied Optics, 54(2), 189-194 (2015).

[11] Press, W.H., Teukolsky, S.A., Vetterling, W.T., and Flannery, B.P., [Numerical Recipes in C], 2nd Ed., Cambridge University Press, (1992). 